\title{
Implementation of NN Controlled DVR for Enhancing the Power Quality by Mitigating Harmonics
}

\author{
P.Abirami ${ }^{1}$, Merin Lizbeth George ${ }^{2}$ \\ Departement of Electrical and Electronics Engineering, Sathyabama Institute of Science and Technology, India
}

\begin{tabular}{l}
\hline Article Info \\
\hline Article history: \\
Received Dec 22, 2017 \\
Revised Jan 11, 2018 \\
Accepted Jan 31, 2018 \\
\hline
\end{tabular}

\section{Keyword:}

DVR

Harmonics

Neural Network Controller

Power Quality

THD

\begin{abstract}
Now a days there is a widespread use of semiconductor devices, which are mostly implemented as the power switches for converters and inverters. These converters and inverters play a vital role in power systems both in transmission and distribution systems. This provides a way for the introduction of harmonics in the power system which leads to poor power quality. To overcome this many solutions have been suggested by the research community but each solution holds its own merits and demerits. Of all these suggested solutions, the Dynamic Voltage Restorer is one of the most cost effective systems for various power quality issues. In this paper the DVR is considered for enhancing the power quality by reducing the harmonics generated because of sensitive loads. Here the power quality is enhanced by controlling the DVR using Neural Network Controller which is trained by Levenberg Marquardt algorithm. In this paper the THD analysis of the voltage quantity is analysed by introducing an unbalanced three phase fault in the system. The simulation is done by using MATLAB/Simulink. From the results, it is verified that the harmonics are reduced by the NN controlled DVR unit. Also the simulation results are verified with the hardware results.
\end{abstract} All rights reserved.

\section{Corresponding Author:}

P.Abirami,

Departement of Electrical and Electronics Engineering,

Sathyabama Institute of Science and Technology, India.

Email: abiramiramkumar80@gmail.com

\section{INTRODUCTION}

There occurs a power quality problem in a power system which possess a non-standard voltage, current and frequency. This results in the economic crisis at consumer's premises and also to the manufacturers of electrical equipment. Industrial automation includes major applications of power electronic systems [1]. These power electronic systems are sensitive to disturbances and become less tolerant to power quality problems such as voltage sags, swells and harmonics. In this paper Dynamic voltage restorer technique is used to mitigate the harmonics produced due to the sensitive load. Dynamic voltage restorers (DVRs) are now becoming more established in industry to reduce the impact of voltage sags to sensitive loads. However, DVRs spend most of their time in standby mode, since voltage sags occur very infrequently, and hence their utilization is low [2]. So, the DVR can be utilised to compensate for the reduction of harmonics in the power system. Thus the power quality of the system can be improved to achieve the satisfaction of the consumers. Dynamic Voltage Restorer (DVR) is a most cost effective and efficient approach to improve the voltage quality at load side. DVR is a power electronic converter based Distributed- 
Static Synchronous Series Compensator (DSSSC), designed to protect the sensitive load [3]. It is connected in series with distribution network, which maintains the sensitive load voltage which can be adjusted for its phase, Magnitude and frequency [4]. Here the voltage is injected by the DVR through an injection transformer and an LC filter and hence the DVR unit is connected in series with the sensitive load. Thus DVR is considered as a voltage restorer which helps in enhancing the power quality of the system [5 - 8]. Thus a required voltage with desired magnitude and phase angle is injected into the system to improve the power quality. Also it helps in restoring the balanced and sinusoidal voltage of the system under the distorted conditions [9].

\section{NEURAL NETWORK}

In our day to day life we are searching for advanced technolofies for improving our activities. To achieve this many researchers have computed soft computing techniques like Fuzzy, ANFIS, ANN controlled systems etc., Of all these techniques, in this paper NN controller is considered for controlling the DVR to supply compensated voltage to the sensitive loads. Neural networks includes the training of neurons to attain a specific charateristics. A neuron is nothing but derived from the studies of human brain neurons. A neuron structure consists of input (dentrites) and an output (Axon) to communicate with the neighbour neurons. By the influence of these dentrites and axon basic structure for Neural network is developed [10]. Before connecting the NN controller in the system, the neurons should be trained effectively by using some basic algorithms.

Neural networks can be trained in their platform to provide better performance. They can be utilised to approximate some of the smooth non-linear functions and hence they are named as universal approximators. For building an efficient neural nets, it has to be learned and trained properly by the experts. Neural network training can be performed either in offline or online mode [11 - 12]. A variety of algorithms are existing to train a neural network controller. Here Levenberg marquardt algorithm is considered for training the neural nets. The LM method is also known as Damped Least Squares Methods (DLS) to solve non-linear least squares problems. This is the most widely used optimization algorithm for the non-linear least squares problems [13]. It possess the advantages of both gradient-descent and Gauss-Newton methods.

Figure 1 represents the Neuron structure of the levneberg marquardt algorithm for training the neurons in the neural network controller. Here the structure consists of two input layers (Reference Input and Actual Input) and ten hidden layers and one output layer (Duty Ratio). The Neurons in this algorithm are trained based on the performance of the system and the trained unit is connected with the DVR unit to improve its performance to reduce the harmonics to obtain the stability of voltage in the system which in turn enhances the power quality of the system [14]. As said before the harmonics are injected into the system beacause of non-linear loads. Thus the system is controlled by a trained NN controller to improve the quality of the system by mitigating the harmonics propagated in the distribution networks [15-17].

Figure 2 gives the inferences about the performance curve of the trained Neural Network controller. The best validation performance is obtained at epoch 1 and the neurons are trained to this best fit line. These neurons are trained by Levenberg Marquardt Algorithm. After that the trained neurons are used to form a neural network structure with input, hidden and output layers and these structure is used as a controller to control the DVR unit for its better performance. Figure 3 represents the Neural Network block of levenberg Marquardt algorithm. Here there are two input layers which represents the reference input and actual input, ten hidden layers and one output layer which represents the duty ratio of the system for generating the pulses for the switches in the converters.

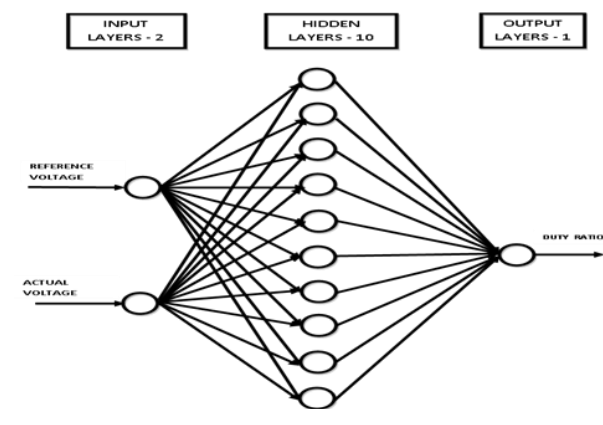

Figure 1. Neuron Structure of Levenberg Marquardt Algorithm

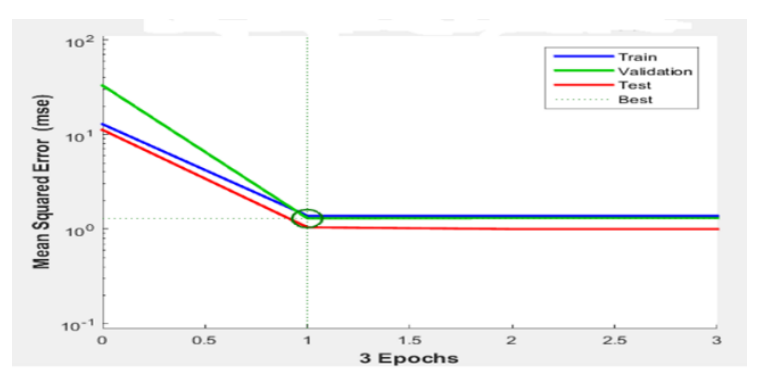

Figure 2. Performance Curve for Trained Neurons of Levenberg Marquardt Algorithm 


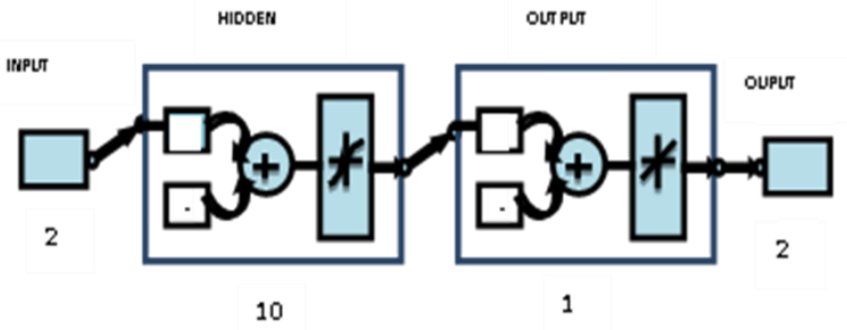

Figure 3. Neural Network Block for Levenberg Marquardt Algorithm

\section{SIMULATION RESULTS AND DISCUSSIONS}

Figure 4 represents the simulation diagram for Neural Network controlled DVR unit for reducing harmonics due to sensitive loads. It consists of DVR unit which is controlled by the Neural Network Controller. The output of DVR is fed into the transmission line i.e., a counter voltage is fed to the line to compensate for the voltage drop in the line due to the generation of harmonics because of sensitive loads. The line voltage is distracted by giving a unbalanced three phase fault on the load side of the system.

Figure 5 represents the harmonic voltage of the system which is created by introducing a three phase fault on the load side. Here the three phase voltages are having different frequencies and amplitude. Figure 6 represents the output voltage of the system after the harmonics have been reduced by the introduction of DVR unit. Here all the three phase voltages have same magnitude and amplitude. From the diagram it is clear that the effective removal of harmonics is obtained in this system. Figure 7, 8, 9 represents the FFT analysis of the voltage waveform before mitigating the harmonics for all the three phases (A,B,C). In Phase A the Total HarmonicDistortion is measured as $3.80 \%$. In Phase B its value is $0.26 \%$ and in $\mathrm{C}$ it is observed as $0.33 \%$. Figure 10, 11, 12 represents the FFT analysis of voltage quantity for three phases $(\mathrm{A}, \mathrm{B}, \mathrm{C})$ whose THD value have been improved. For phase A the Total Harmonic Distortion is $0.09 \%$. For phase B it is observed as $0.13 \%$ and for phase $\mathrm{C}$ it is obtained as $0.17 \%$

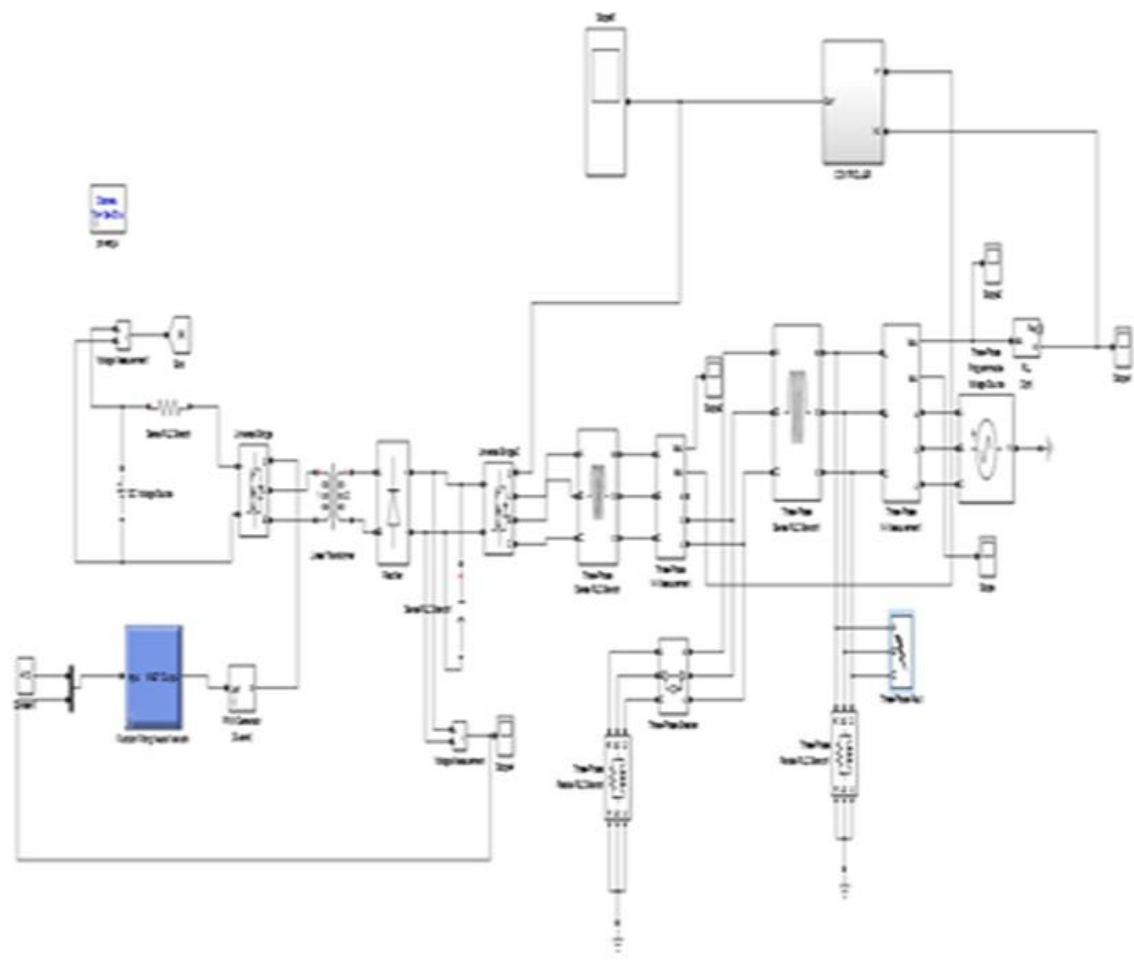

Figure 4. Simulation Diagram for Neural Network Controlled DVR Unit for Harmonics Reduction 


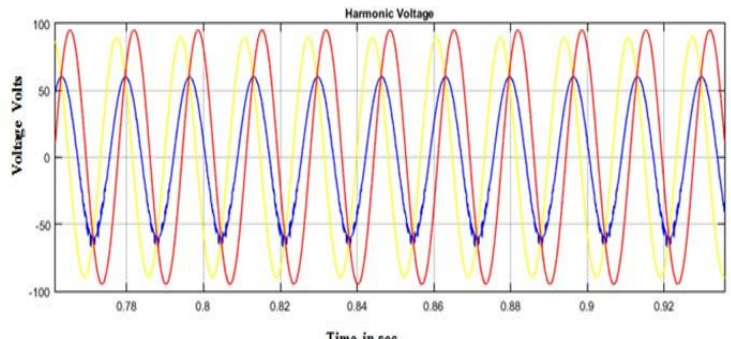

Figure 5. Harmonic Voltage Due to Three Phase Fault Created on The Load Side.

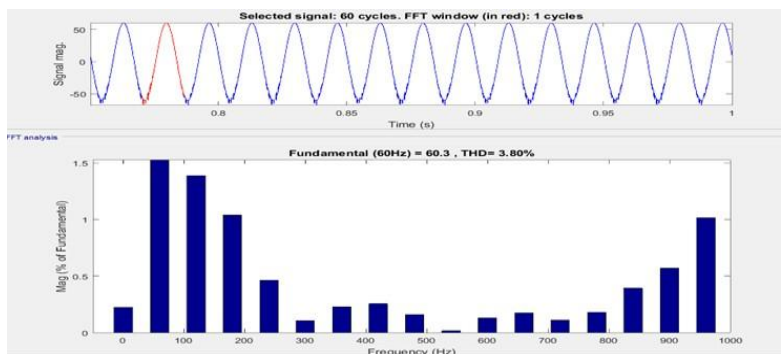

Figure 7. FFT Analysis for Phase A Before Mitigating Harmonics

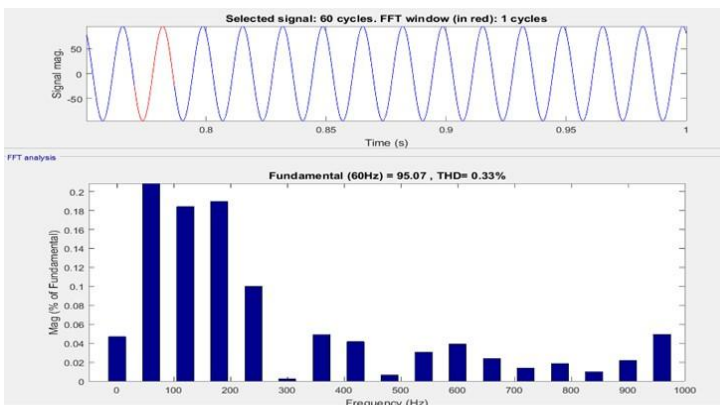

Figure 9. FFT Analysis for Phase C Before Mitigating Harmonics

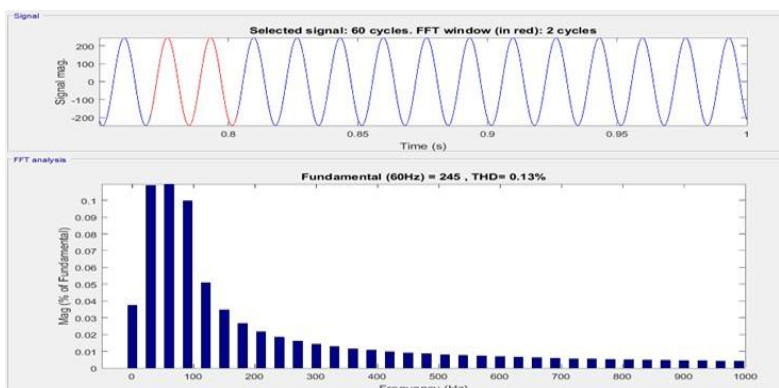

Figure 11. FFT Analysis for Phase B After the Removal of Harmonics

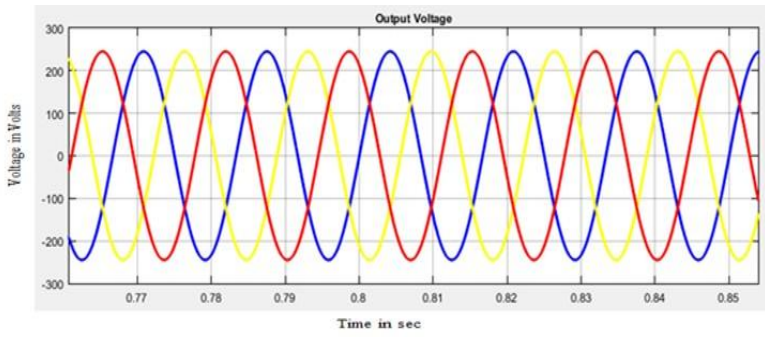

Figure 6. Output Voltage of The System After the Removal of Harmonics.

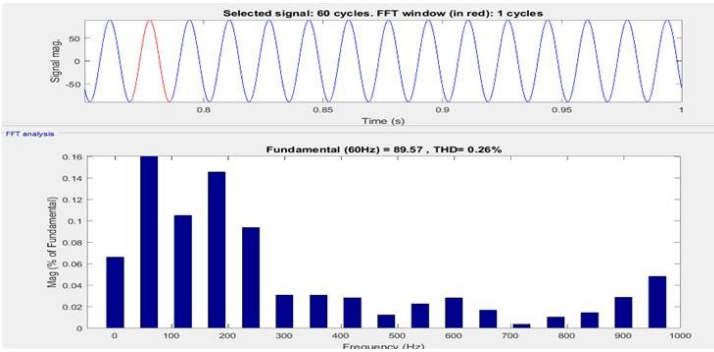

Figure 8. FFT Analysis for Phase B Before Mitigating Harmonics

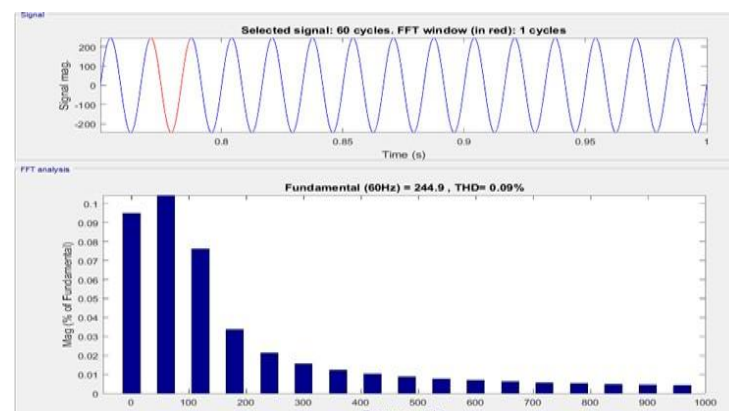

Figure 10. FFT Analysis for Phase A After the Removal of Harmonics

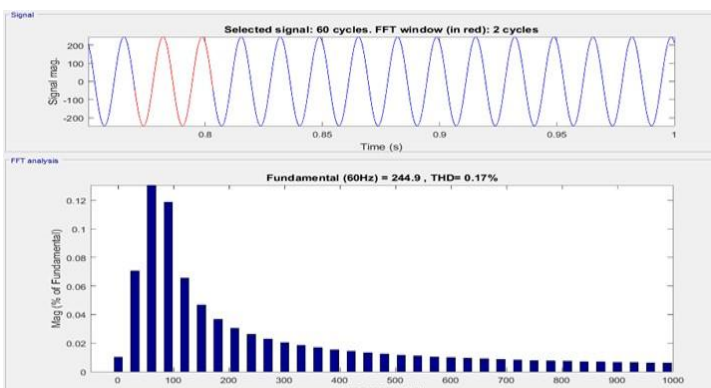

Figure 12. FFT Analysis for Phase C After the Removal of Harmonics 
Table 1 gives details about the THD value before reducing the harmonics for different phases of the system. Table 2 gives details about the THD value after reducing the harmonics for different phases of the system.

Table-1 THD Before Reducing Harmonics

\begin{tabular}{lll}
\hline S.No & Phases & \% THD \\
\hline 1 & Phase A & 3.80 \\
2 & Phase B & 0.26 \\
3 & Phase c & 0.33
\end{tabular}

Table-2 THD after reducing harmonics

\begin{tabular}{lll}
\hline S.No & Phases & \% THD \\
\hline 1 & Phase A & 0.09 \\
2 & Phase B & 0.13 \\
3 & Phase c & 0.17 \\
\hline
\end{tabular}

\section{HARDWARE RESULTS AND DISCUSSIONS}

Figure 13 represents the hardware diagram of DVR unit for reduction of harmonics. It consists of MOSFET Driver circuit which carries the MOSFET switches for the converters. A NN based controller for driving the DVR. A insulation transformers for protecting the control circuitry from the power circuitry and a step down transformer for reducing the supply voltage $230 \mathrm{~V}$ to $120 \mathrm{~V}$. It also consists of a Voltage Source Inverter which acts as a main component of the DVR unit. It consists of a driver circuit board for driving the MOSFET switches. This hardware module is designed based on the simulation results obtained from the software. Figure 14 represents the voltage waveform of the power system before the reduction of harmonics. Figure 15 gives the output voltage of the hardware unit after the removal of harmonics from the system.

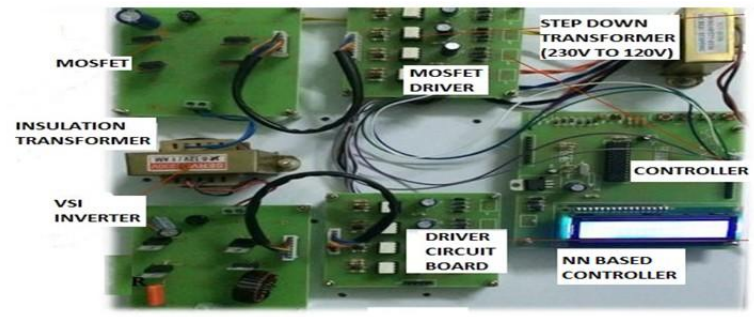

Figure 13. Hardware of DVR Unit for Reduction of Harmonics

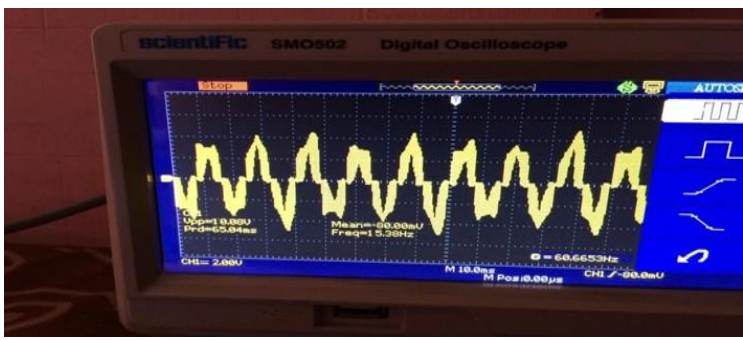

Figure 14. Voltage Waveform of the Hardware Unit Before Compensation for Harmonics.

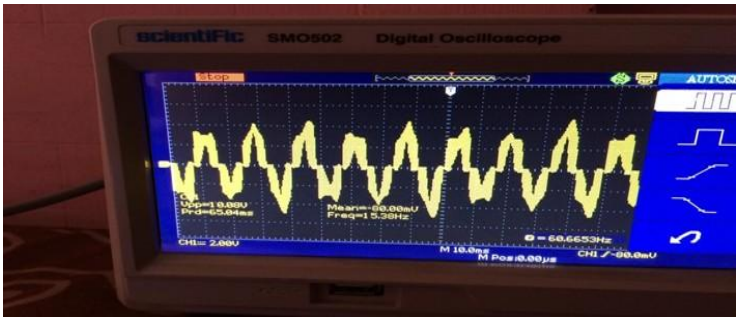

Figure 14. Voltage Waveform of the Hardware Unit Before Compensation for Harmonics

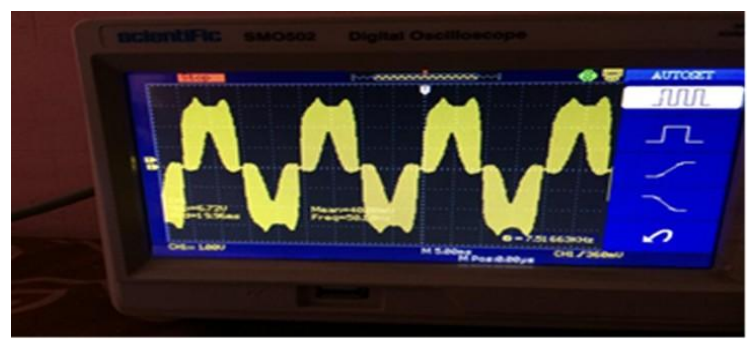

Figure 15. Output Voltage Waveform of the Hardware Unit After Compensation for Harmonics

\section{CONCLUSION}

Dynamic Voltage restorer is an efficient system which is applied to compensate for power quality issues like Voltage Sag/Swell And harmonics that occurs due to external disturbances. As the external disturbances are due to natural calamities, most of the time the DVR is under Static condition while it is used for compensating the voltage sag/swell of the system. So, it can be efficiently used for harmonics compensation due to sensitive loads. In this paper the harmonics of the power system due to sensitive loads are rectified by using a NN controlled DVR unit. The Levenberg marquardt algorithm for trainig the NN 
controller is explained here. A non-linear three phase fault is introduced into the system to deal about the harmonics generation and compensation for the harmonics due to sensitive loads by DVR. The THD amnalysis for before and after compensation for harmonics is anlysed. From the simulation results and FFT analysis it is obvious that the harmonics of the system has been reduced. The hardware is also made for the system to perform the harmonic analysis. Thus the THD and the distortion in the load voltage are reduced with the application of NN controlled DVR. From the above results it is clear that the proposed system provides better mitigation of harmonics due to sensitive loads.

\section{REFERENCES}

[1] Sandesh Jain, Prof.Shivendra Singh Thakur, Prof. S.P.Phulambrikar, "Fuzzy Controller Based DVR To Mitigate Power Quality And Reduce The Harmonics Distortion Of Sensitive Load" International Journal of Advanced Research in Electrical, Electronics and Instrumentation Engineering Vol. 1, Issue 5, November 2012

[2] Michael John Newman, Member, IEEE, Donald Grahame Holmes, Senior Member, IEEE, John Godsk Nielsen, Member, IEEE, and Frede Blaabjerg, Fellow, IEEE “A Dynamic Voltage Restorer (DVR) With Selective Harmonic Compensation at Medium Voltage Level", IEEE transactions on industry applications, vol. 41, no. 6, november/december 2005

[3] Sathish Babu Pandu and Kamaraj Nagappan “A Novel Multiobjective Control of DVR to Enhance Power Quality of Sensitive Load” Hindawi Publishing Corporation Scientific World Journal Volume 2015.

[4] K.Surendar, M.Ramya, M.Muruganandam, "Control of Reduced Rating Dynamic Voltage Restorer With Energy Storage System using Fuzzy Logic" International Journal of Innovative Research in Science, Engieering and Technology,Vol.4, Special Issue6, May 2015.

[5] Walid Frangieh, Maged B.Najjar "Active control for power quality improvement in hybrid power systems" IEEE Xplore Technological June 2015

[6] Krischonme Bhumkittipich and Nadarajah Mithulananthan "Performance Enhancement of DVR for Mitigating Voltage Sag/Swell using Vector Control Strategy” Elsevier Energy Procedia 9 ( 2011 ) 366 - 379.

[7] M. Nabipour n, M.Razaz,S.GH.Seifossadat,S.S.Mortazavi "A novel adaptive fuzzy membership function tuning algorithm for robust control of a PV-based Dynamic Voltage Restorer (DVR)" Elsevier 2016 Engineering ApplicationsofArtificial Intelligence53(2016)155-175.

[8] C.K. Sundarabalan $\Uparrow, K$. Selvi "Compensation of voltage disturbances using PEMFC supported Dynamic Voltage Restorer” Elsevier Electrical Power and Energy Systems 71 (2015) 77-92.

[9] K. Chandrasekaran , V.K. Ramachandaramurthy "An improved Dynamic Voltage Restorer for power quality improvement” Elsevier 2016 Electrical Power and Energy Systems 82 (2016) 354-362.

[10] Jürgen Schmidhuber "Deep learning in neural networks: An overview" Elsevier 2015.

[11] Lester S. H. Ngia, Jonas Sjöberg, "Efficient Training of Neural Nets for Nonlinear Adaptive Filtering Using a Recursive Levenberg-Marquardt Algorithm” IEEE Transactions On Signal Processing, Vol. 48, No. 7, July 2000.

[12] G. Lera and M. Pinzolas "Neighborhood Based Levenberg-Marquardt Algorithm for Neural Network Training" IEEE Transactions On Neural Networks, Vol. 13, No. 5, September 2002.

[13] Kit Yan Chan, Tharam S. Dillon, Jaipal Singh, and Elizabeth Chang "Neural-Network-Based Models for ShortTerm Traffic Flow Forecasting Using a Hybrid Exponential Smoothing and Levenberg-Marquardt Algorithm" IEEE Transactions On Intelligent Transportation Systems, Vol. 13, No. 2, June 2012

[14] R. Balasubramanian, S. Palani, "Simulation And Performance Evaluation Of Shunt Hybridpower Filter For Power Quality Improvement Using PQ Theory" International Journal of Electrical and Computer Engineering" Vol.6, No.6 December 2016 pp2603-2609.

[15] M. Jawad Ghorbani*, H. Mokhtari** “ Impact of Harmonics on Power Quality and Losses in Power Distribution Systems" International Journal of Electrical and Computer Engineering, Vol. 5, No. 1, February 2015, pp. 166 174

[16] Jarupula Somlal, Venu Gopala Rao.Mannam, Narsimha Rao.Vutlapalli "Power Quality Improvement in Distribution System using ANN Based Shunt Active Power Filter" International Journal of Power Electronics and Drive System Vol. 5, No. 4, April 2015, pp. 568 575

[17] G. Ramya , V. Ganapathy , P. Suresh "Power Quality Improvement Using Multi-level Inverter based DVR and DSTATCOM Using Neuro-fuzzy Controller" International Journal of Power Electronics and Drive System (IJPEDS) Vol. 8, No. 1, March 2017, pp. 316 324 